\title{
Charge-tunable graphene dispersions in water made with amphoteric pyrene derivatives
}

DOI:

10.1039/C9ME00024K

\section{Document Version}

Accepted author manuscript

Link to publication record in Manchester Research Explorer

\section{Citation for published version (APA):}

Shin, Y., Just-baringo, X., Zarattini, M., Isherwood, L., Baidak, A., Kostarelos, K., Larrosa, I., \& Casiraghi, C. (2019). Charge-tunable graphene dispersions in water made with amphoteric pyrene derivatives. Molecular Systems Design \& Engineering. https://doi.org/10.1039/C9ME00024K

\section{Published in:}

Molecular Systems Design \& Engineering

\section{Citing this paper}

Please note that where the full-text provided on Manchester Research Explorer is the Author Accepted Manuscript or Proof version this may differ from the final Published version. If citing, it is advised that you check and use the publisher's definitive version.

\section{General rights}

Copyright and moral rights for the publications made accessible in the Research Explorer are retained by the authors and/or other copyright owners and it is a condition of accessing publications that users recognise and abide by the legal requirements associated with these rights.

\section{Takedown policy}

If you believe that this document breaches copyright please refer to the University of Manchester's Takedown Procedures [http://man.ac.uk/04Y6Bo] or contact uml.scholarlycommunications@manchester.ac.uk providing relevant details, so we can investigate your claim.

\section{OPEN ACCESS}




\section{MSDE IChemE}

\section{Charge-tunable graphene dispersions in water made with amphoteric pyrene derivatives}

\begin{tabular}{|c|c|}
\hline Journal: & Molecular Systems Design \& Engineering \\
\hline Manuscript ID & ME-ART-02-2019-000024.R1 \\
\hline Article Type: & Paper \\
\hline $\begin{array}{r}\text { Date Submitted by the } \\
\text { Author: }\end{array}$ & 02-Apr-2019 \\
\hline Complete List of Authors: & $\begin{array}{l}\text { Shin, Yuyoung; University of Manchester, School of Chemistry } \\
\text { Just-Baringo, Xavier; University of Manchester, School of Chemistry } \\
\text { Zarattini, Marco; University of Manchester, School of Chemistry } \\
\text { Isherwood, Liam; University of Manchester, School of Chemistry; } \\
\text { University of Manchester, Dalton Cumbrian Facility } \\
\text { Baidak, Aliaksandr; University of Manchester, Dalton Cumbrian Facility; } \\
\text { University of Manchester, School of Chemistry } \\
\text { Kostarelos, Kostas; University of Manchester, Nanomedicine Lab, School } \\
\text { of Medicine \& National Graphene Institute } \\
\text { Larrosa, Igor; University of Manchester, School of Chemistry } \\
\text { Casiraghi, Cinzia; University of Manchester, School of Chemistry }\end{array}$ \\
\hline
\end{tabular}

\section{SCHOLARONE ${ }^{m}$ \\ Manuscripts}




\title{
Charge-tunable graphene dispersions in water made with amphoteric pyrene derivatives
}

Yuyoung Shin a, Xavier Just-Baringo ${ }^{a}$, Marco Zarattinia, Liam H. Isherwooda,b, Aliaksandr Baidak ${ }^{a, b}$, Kostas Kostarelos ${ }^{c}$, Igor Larrosa*a, and Cinzia Casiraghi*a

a School of Chemistry, University of Manchester, Oxford Road, Manchester, United Kingdom, M13 9PL

${ }^{b}$ Dalton Cumbrian Facility, University of Manchester, Westlakes Science and Technology Park, Moor Row, Cumbria, United Kingdom, CA24 $3 \mathrm{HA}$

cNanomedicine Lab, Faculty of Biology, Medicine and Health, AV Hill Building, University of Manchester, Manchester, United Kingdom, M13 9PT

\begin{abstract}
Liquid-phase exfoliation (LPE) of graphite shows great potential as mass scalable and low-cost method for production of solution-processed graphene, which can be used for a wide range of applications. Due to the hydrophobic nature of graphene, a stabilising agent is needed to exfoliate graphite in water - in particular, pyrene derivatives have been shown to be very effective at exfoliating graphite, by producing either positively or negatively charged dispersions, depending on the functional group used.

In this work we have synthesised amphoteric amino acid-based pyrene derivatives, using amino acid-based functional groups (lysine and taurine), and tested them as exfoliating agent for the LPE of graphene. Atomic Force Microscopy shows the flakes to have average size between $100-300 \mathrm{~nm}$, while qualitative Raman analysis shows the dispersion to be composed mainly by single and few-layer flakes, as also confirmed by Transmission Electron Microscopy. Finally, we demonstrate that the surface charge of graphene can be adjusted by a systematic change of the $\mathrm{pH}$ level. Although both stabilisers demonstrated to be able to exfoliate graphite in water, pyrene-based lysine produces more concentrated and stable graphene dispersion, whose surface charge changes between positive to negative depending on $\mathrm{pH}$ level. In contrast, sedimentation of the dispersed material was observed at extreme $\mathrm{pH}$ for graphene dispersions obtained with pyrene-substituted taurine. This is attributed to the low $\mathrm{pKa}$ of the sulfonic group in taurine, which remains negatively charged even at very low $\mathrm{pH}$.
\end{abstract}

\section{Design, System, Application}

Graphene, a 2-dimensional crystal made of $s p^{2}$ carbon atoms arranged in a hexagonal lattice, has shown great potential because of its exceptional properties. One of the challenges of using graphene for biomedical applications is the need to stabilise graphene in an aqueous medium, without compromising its outstanding properties. Pyrene derivatives, such as 1-pyrenesulfonic acid sodium salt, have demonstrated excellent exfoliating and stabilising abilities, by allowing producing highly concentrated and stable graphene dispersions in water. Here, we have synthesised pyrene derivatives with biomolecular functional groups, such as lysine and taurine, to provide biocompatibility and amphoteric properties that allow control of the surface charge. These pyrene derivatives were used for producing stable graphene dispersion in water, whose charge can be tuned by changing the $\mathrm{pH}$ level. This tunable surface charge can be utilised to enhance and/or reduce interaction with specific molecules of interest in future applications. 


\section{Introduction}

Since the discovery of graphene ${ }^{1}$, a single layer of graphite, research on this advanced material has significantly expanded in many fields ${ }^{2,3}$, owing to its exceptional properties, such as high electronic and thermal conductivities, large specific surface area, and remarkable mechanical and chemical stability ${ }^{3}$. Currently, there is the need to develop mass-scalable, simple, low cost, highly efficient and environmental-friendly techniques for the production of graphene.

Liquid phase exfoliation (LPE) marked a decade of research as a mass-scalable and versatile technique to produce graphene and other two-dimensional materials in solution. ${ }^{4-7}$ LPE can be performed in different ways: with bath or tip- sonication, shear mixing and ball milling and microfluidization to produce solution-processed graphene. 4,7-11 Owing to its mass-scalability and versatility, graphene produced by LPE has shown great potential in many applications, including biological and medical applications, such as flexible biomedical electronic devices, biomedical imaging, and gene and drug delivery. ${ }^{12-18}$

In particular, when focusing on biological applications, water is the obvious solvent choice. However, graphene is not dispersible in water. ${ }^{4,19}$ Thus, one of the most popular methods to produce graphene for biomedical applications is based on the alteration of its surface properties, i.e. oxidation of graphene to produce graphene oxide (GO), which significantly enhances the dispersibility of graphene in water, but it also disrupts its conjugated $\pi$-system. ${ }^{20}$ Although GO offers high hydrophilicity and potential of using the oxygen groups for further functionalisation tailored to specific applications, its production involves use of toxic chemicals and timeconsuming processing for purification. ${ }^{21}$ On the other hand, non-covalent functionalisation, based on the use of amphiphilic molecule, has shown to be an effective method to produce stable aqueous solutions of defects-free graphene nanosheets. ${ }^{22-24}$ In particular, pyrene derivatives have shown to produce concentrated and stable dispersions, ${ }^{24-29}$ owing to their $\pi-\pi$ interaction with graphene, and relatively good solubility in water due to the sulfonic group. In particular, previous works from our group ${ }^{24,26,27}$ have demonstrated efficient exfoliation using 1-pyrenesulfonic acid sodium salt to produce stable, concentrated and biocompatible dispersions of graphene. For biomedical application, it is of vital importance to understand the properties of graphene and its effects in a biological environment. It has been shown $n^{12,21,30,31}$ that the properties of graphene, such as lateral size and thickness, type and degree of functionalisation, and surface charge, can significantly influence the biological interaction of graphene, including its toxicity, bio-distribution, degradation, and biotransformation. In particular, the charge on the graphene surface could be utilized to control its interaction with other biomolecules, either by enhancing or reducing its interaction with specific types of biomaterials or biosystems, ${ }^{12,32,33}$ or used for effective drug delivery ${ }^{34}$. Therefore, it is important to test and compare negatively- and positively-charged graphene dispersions. Currently, both GO and oxygen-free graphene dispersions produced with 1pyrenesulfonic acid sodium salt are negatively charged. Thus, further functionalisation is needed to change the sign of the charge. A few studies have also reported the use of other types of pyrene derivatives, such as amino pyrene or pyrenyliminolipids, to produce positively charged graphene, ${ }^{25,29,35}$ often resulting in low-concentrated and/or unstable graphene dispersions, which therefore cannot be exploited for biological applications.

In this work we propose an alternative approach, based on the use of a charge-tunable stabiliser, thus allowing for direct comparison of biological effects associated to the type of charge. To the best of our knowledge, only one approach has been reported so far to produce charge-tunable graphene dispersions for biological applications. This is based on the use of bovine serum albumin (BSA). ${ }^{36,37}$ The tunable surface charge is due to the amphoteric nature of BSA; however, graphene exfoliation is not very efficient, as dispersions mostly contain thick graphene layers. ${ }^{36,37} \beta$-lactoglobulin, ovalbumin, lysozyme, and hemoglobin have been also tested, but BSA was reported to have the highest exfoliation efficiency amongst all. ${ }^{37,38}$

In this work, we designed a new family of pyrene-based stabilisers, composed of a pyrene base linked to amino acids, lysine (py-lys) or taurine (py-tau), for efficient production of stable aqueous dispersion of graphene with tunable surface charge. The smaller size, simple structure and aromaticity of pyrene-based stabilisers allow improved interaction with graphene, compared to BSA, and therefore better exfoliation efficiency. Rather than using a protein containing a large number of amino acids, our approach is based on the use of a simple pyrene derivative, which is functionalised with a single amino acid. Furthermore, our approach avoids problems related to the structure or folding of the protein on the exfoliation efficiency. ${ }^{36,37}$ The amphoteric pyrene derivatives are used to produce stable graphene dispersions in water by LPE. The material is characterised by using different techniques: the concentration of the dispersions is determined by UV-Vis spectroscopy, while thickness and lateral size distribution of the exfoliated graphene nanosheets are characterised by Raman spectroscopy, Atomic Force Microscopy (AFM), and transmission electron microscopy (TEM). Zeta potential (ろ) is used as a measure of surface charge of graphene nanosheets. Here, the change of surface charge of graphene is studied by monitoring

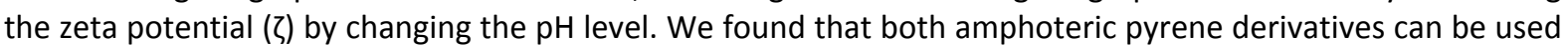


for graphene exfoliation, although dispersions obtained with py-lys showed higher concentration and better stability, at both high and low pH levels. Thus, we demonstrated a simple method to obtain charge-tunable aqueous graphene dispersions with potential for use in biological applications.

\section{Experimental Section \\ Synthesis}

\section{Synthesis of pyrene derivatives with amphoteric functional groups}

For better biocompatibility of the produced graphene dispersion, naturally occurring biomolecular functional groups were chosen: lysine and taurine, which are amino acids, the building blocks of proteins, with amphoteric nature. The pyrene-lysine adduct (py-lys, in the Supporting Information) was prepared using 1-pyrenylacetic acid (S2 in the SI, Sigma-Aldrich) and an adequately protected lysine derivative (S1 in the SI). The amide bond formation was performed using hydroxybenzotriazole/1,3-diiscaropropylcarbodiimide (HOBt/DIC, Fisher Scientific) as the coupling reagents and the resulting products were deprotected with trifluoroacetic acid (TFA, Sigma-Aldrich) to yield the corresponding unprotected product as TFA salt. The desired product was obtained as a free-flowing solid after precipitation in ether. The pyrene-taurine adduct (py-tau in the SI) was obtained by reductive amination between commercially available 1-pyrenecarboxaldehyde (Sigma-Aldrich) and taurine (Sigma-Aldrich) with sodium borohydride as the reducing agent. The desired product was obtained after recrystallisation in $\mathrm{CH}_{2} \mathrm{Cl}_{2}$. Further synthesis and characterisation details of materials are available in the Supporting Information.

\section{Preparation of graphene dispersions}

Graphene dispersions were prepared via LPE of graphite in water. In details, $3 \mathrm{mg} \cdot \mathrm{ml}^{-1}$ of graphite (Graphexel Itd.) was added to $100 \mathrm{ml}$ of de-ionized (DI) water, previously mixed with $0.4 \mathrm{mg} \cdot \mathrm{ml}^{-1}$ of pyrene derivatives (pylys or py-tau). The mixture was then sonicated at 600W using a Hilsonic bath sonicator for 7 days at constant temperature. Afterwards, un-exfoliated graphite was removed by 2-steps centrifugation (Sigma 1-14k refrigerated centrifuge) at $3500 \mathrm{rpm}(903 \mathrm{~g})$ for 20 minutes. After each centrifugation steps, the supernatant containing graphene and the pyrene derivatives in water was collected. The corresponding graphene dispersions produced with py-lys and py-tau are called Gr/py-lys and Gr/py-tau, respectively.

\section{Characterization}

\section{UV-Vis Spectroscopy}

The final concentration of graphene is determined by UV-Vis spectroscopy. The UV-Vis spectrum of graphene appears flat and featureless in the visible-IR region, ${ }^{4}$ so the absorption is measured at $660 \mathrm{~nm}$ for estimation of the graphene nanosheet concentration using the Beer-Lambert law. ${ }^{39}$ An absorption coefficient of $2460 \mathrm{~L} \cdot \mathrm{g}^{-}$ ${ }^{1} \cdot \mathrm{m}^{-1}$ is used for estimating the graphene concentration.6,22,40 A Perkin-Elmer I-900 UV-Vis-NIR spectrophotometer was used to acquire the spectra.

\section{Zeta-Potential Measurements}

Electrophoretic mobility $(\mu)$ was measured using a ZetaSizer Nano ZS (Malvern Instruments, UK) after dilution of samples with water in the folded capillary cells (Malvern Instruments, UK). Default instrument settings for water-based system and automatic analysis were used for all measurements, performed at $25^{\circ} \mathrm{C}$ and at the

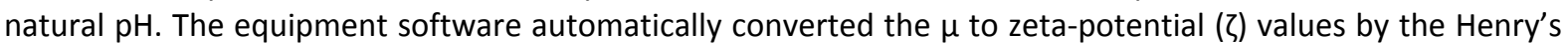
equation ${ }^{41}: \mu=2 \varepsilon \zeta \mathrm{F}(\mathrm{ka}) / 3 \eta$ where $\varepsilon$ is dielectric constant, $\eta$ is the solution viscosity and $F(\kappa a)$ is the Henry's function which is approximated to the value of 1.5 using Smoluchowski approximation for polar media, valid for dispersed particles of any shape including platelet-like particles. All values for samples are mean $\pm S D$, calculated from triplicate measurements.

\section{pH Measurement}

$\mathrm{pH}$ measurements of graphene dispersions were performed with a bench top $\mathrm{pH}$ meter (Mettler Toledo Seven Easy S20, US) equipped with a pH electrode integrated with temperature probe (InLabExpert Pro). Adjustment of $\mathrm{pH}$ of graphene dispersions, ranged between 1.0 and 13.0, was made by using concentrated $\mathrm{HCl}$ and $\mathrm{NaOH}$ solutions to achieve acidic and basic $\mathrm{pH}$ level, respectively. Typically, only a few tens to a few hundred $\mu \mathrm{L}$ of concentrated $\mathrm{HCl}$ and $\mathrm{NaOH}$ solutions were added to $4 \mathrm{ml}$ of graphene dispersions to minimise the change in the graphene concentration.

\section{AFM}

A Bruker Atomic Force Microscope (MultiMode 8) in Peak Force Tapping mode, equipped with ScanAsyst-Air tips was used to determine the lateral size distribution of the flakes. The sample was prepared by drop casting the solution on a clean silicon substrate; several areas of $100 \mu \mathrm{m}^{2}$ were scanned and about 500 flakes were 
selected for lateral size analysis. Lateral dimension and thickness distributions of graphene nanosheets were carried out using Gwyddion scanning probe microscopy data processing software.

\section{Raman Spectroscopy}

Raman measurements were performed using a Renishaw Invia Raman spectrometer equipped with $514.5 \mathrm{~nm}$ excitation line, using $2.0 \mathrm{~mW}$ laser power on the sample. Graphene dispersions were drop cast onto silicon substrates and Raman measurements were taken with a $100 \times(\mathrm{NA}=0.85)$ objective lens and 2400 grooves $/ \mathrm{mm}$ grating. Typically more than 50 Raman spectra were obtained on individual flakes for each sample for statistical analysis.

\section{TEM}

Graphene dispersions were diluted until optically transparent using deionised water. This corresponds to a diluted dispersion concentration of less than $5 \mu \mathrm{g} \cdot \mathrm{mL}^{-1}$ which suppresses flake aggregation during deposition. A 300 mesh Cu TEM grid with a lacey carbon support film (Agar Scientific) was placed onto filter paper and a single drop of diluted dispersion was deposited onto the carbon film. The TEM grid was then dried at $50{ }^{\circ} \mathrm{C}$ under reduced pressure for 30 minutes to remove residual water. Bright field imaging and electron diffraction were carried out using a Tecnai G² 20 S-TWIN Analytical TEM (FEI) operating at $200 \mathrm{kV}$.

\section{Results and discussion}

\section{Exfoliation of graphene and its characterisation}

Aqueous graphene dispersions were prepared by ultrasonication of graphite powder in water mixed with pyrene derivatives (py-lys and py-tau, Figure $1 \mathrm{a}-\mathrm{b}$ ).

(a)

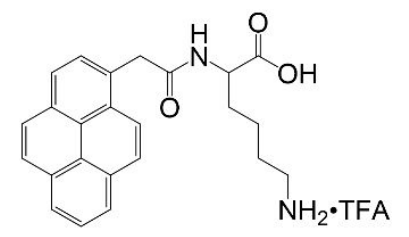

(b)

(c)
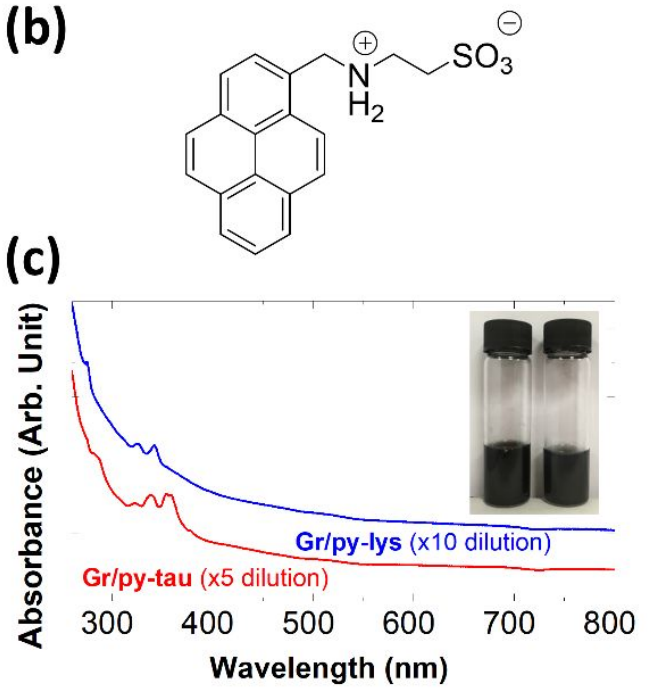

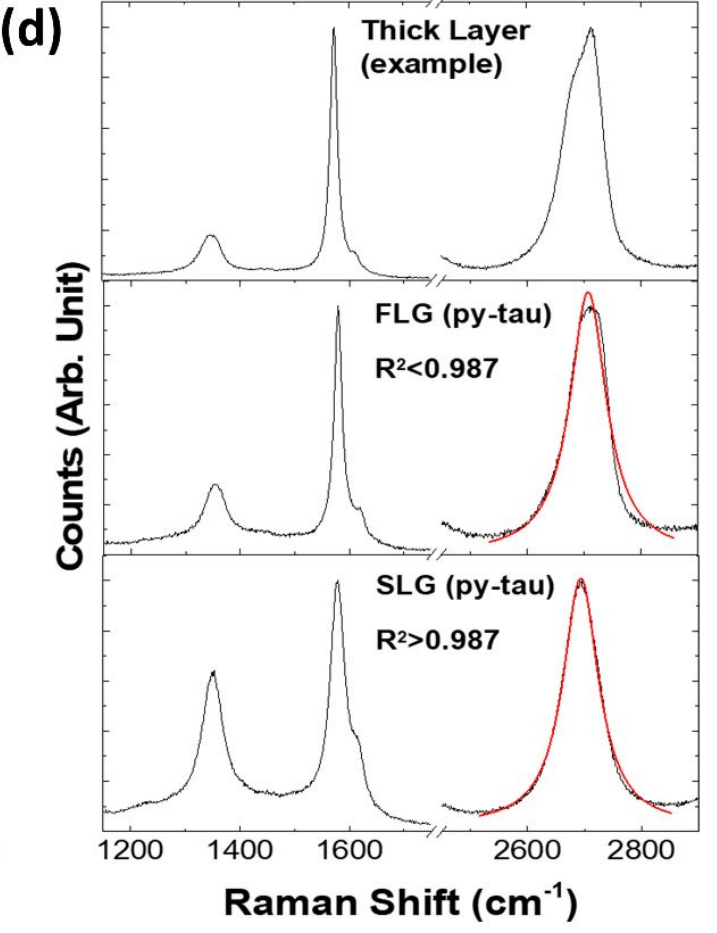

Figure 1 Chemical structure of (a) py-lys and (b) py-tau; (c) UV-Vis spectra of the obtained graphene dispersions. Inset: photograph of (left) Gr/py-lys and (right) Gr/py-tau dispersions; (d) Representative Raman spectra for graphene flakes prepared with py-tau (red curves are the Lorentzian fits of the 2D peaks).

Gr/py-lys Gr/py-tau




\begin{tabular}{|c|c|c|}
\hline $\begin{array}{l}\text { Concentration } \\
\left(\mathrm{mg} \cdot \mathrm{ml}^{-1}\right)\end{array}$ & 0.232 & 0.074 \\
\hline $\mathrm{pH}$ & 3.1 & 4.1 \\
\hline 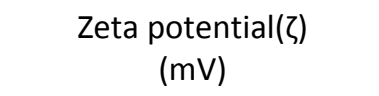 & 22.9 & -30.4 \\
\hline $\begin{array}{l}\text { Average Flake Size } \\
\qquad(\mathrm{nm})\end{array}$ & 221 & 276 \\
\hline $\begin{array}{c}\text { Percentage of single-layer } \\
\text { graphene flakes }\end{array}$ & $\sim 44 \%$ & $\sim 42 \%$ \\
\hline
\end{tabular}

Table 1 Summary of the properties of the graphene dispersions obtained using py-lys and py-tau exfoliating agents.

Figure 1 (c) shows the UV-Vis spectrum of the graphene dispersions: graphene shows flat absorption in visibleIR region with broad absorption peak around $270 \mathrm{~nm}$ corresponding to $\pi-\pi *$ electron transition. ${ }^{42}$ Small pyrene peaks are also observed in the region between $300-400 \mathrm{~nm}$. From the UV-Vis spectra, we calculated a concentration of 0.232 and $0.074 \mathrm{mg} \cdot \mathrm{ml}^{-1}$ for graphene dispersions prepared with py-lys and py-tau, respectively. Thus, the graphene dispersion prepared with py-lys showed higher graphene exfoliation and stabilisation efficiency compared to that obtained using py-tau as exfoliating agent. This could be due to the different distance of the charged functional group from the pyrene base: the more distant the charged functional groups, the better the separation of interactions between pyrene adsorption on graphene surface and solvation of the functional groups within water molecules, as observed for traditional surfactants. ${ }^{43}$ Parviz et al. ${ }^{25}$ observed a similar behavior with carboxylated pyrenes: 1-pyrenebutyric acid showed higher exfoliation efficiency compared to 1-pyrenecarboxylic acid owing to increased distance between the pyrene base and the functional group.

Both of the as-prepared graphene dispersions were acidic, showing $\mathrm{pH}$ of 3.1 and $4.1 \mathrm{for} \mathrm{Gr} / \mathrm{py}$-lys and $\mathrm{Gr} / \mathrm{py}$ tau, respectively. The zeta potential of the as-prepared graphene dispersions are $22.9 \mathrm{mV}$ and $-30.4 \mathrm{mV}$ for $\mathrm{Gr} / \mathrm{py}$-lys and $\mathrm{Gr} / \mathrm{py}$-tau, respectively. The different charge of as-prepared graphene dispersions could be attributed to two major factors: the different $\mathrm{pH}$ levels of the two dispersions and the different acid dissociation constant $(p K a)$ values of the sulfonate group ( $\mathrm{p} K a \approx-7$ ) compared to that of carboxylic acid ( $\mathrm{pKa} \approx+5$ ). Due to the extremely low $\mathrm{pKa}$ value of sulfonate group, negative charge is prevalent for Gr/py-tau, whereas for Gr/pylys, carboxylic acid would not likely to be dissociated at pH 3 in its as-prepared state, while the ammonium group will remain protonated in both compounds at acidic $\mathrm{pH}$.

The exfoliated graphene nanosheets were further characterised by AFM, TEM and Raman spectroscopy for thickness and lateral size measurement. Figure 2(a) and (b) show representative AFM images and the corresponding height profile for representative graphene nanosheets from Gr/py-lys and Gr/py-tau, respectively. For statistical analysis of thickness and lateral size distribution, AFM images of larger area with more than two hundreds of individual flakes were used (Figures S1 and S3). The corresponding histograms are shown in Figures S2 and S4. AFM shows that the average flake size for Gr/py-lys and Gr/py-tau are $221 \mathrm{~nm}$ and $276 \mathrm{~nm}$, respectively, with more than $90 \%$ of the flakes having a size between $50 \mathrm{~nm}$ and $800 \mathrm{~nm}$, which is typical of graphene dispersions obtained by LPE with pyrene derivatives. ${ }^{24,26,27}$ Thickness analysis of graphene flakes from AFM images shows that most of the flakes have a thickness in the range of $2-10 \mathrm{~nm}$. It should be noted that although the theoretical thickness of single-layer graphene is $\sim 0.34 \mathrm{~nm}$, the height measurement by AFM do not provide this thickness for graphene due to the instrumental offset caused by capillary forces or adhesion. ${ }^{44,45}$ This effect is very relevant for graphene produced by LPE as solvent molecules and exfoliating agents are adsorbed on both surfaces. Many studies have reported a measured height of $1-2 \mathrm{~nm}$ for single layer graphene produced by solution-processing. . $, 23,27,46^{2}$ 
(a)
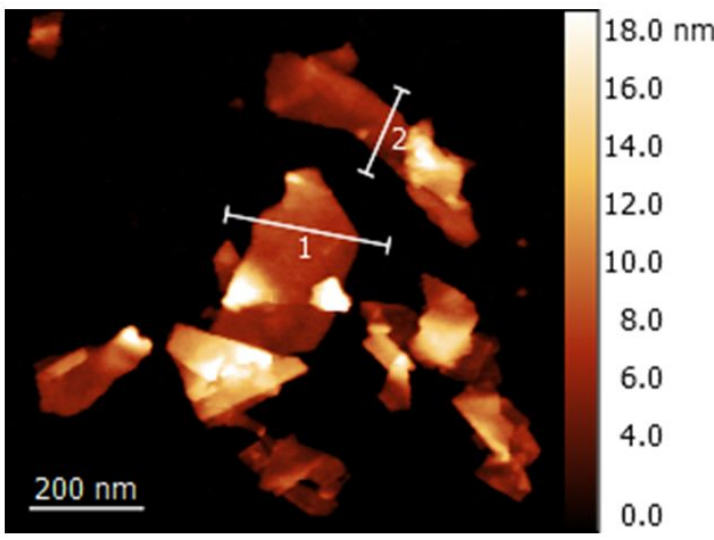

(b)

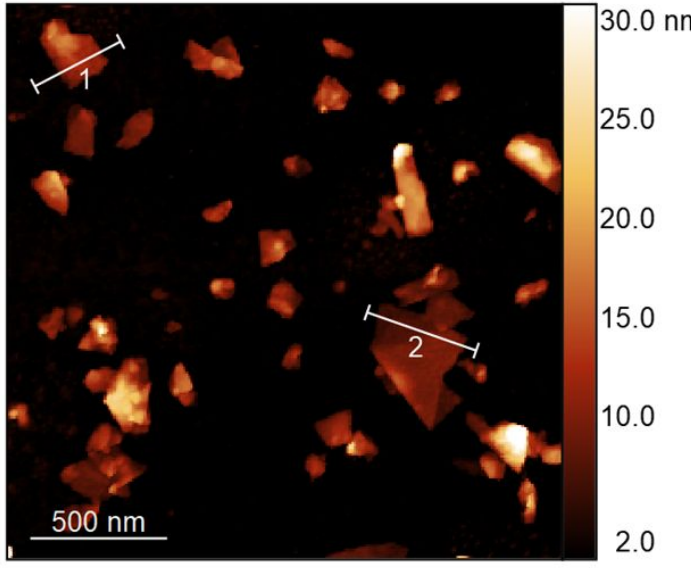

(c)

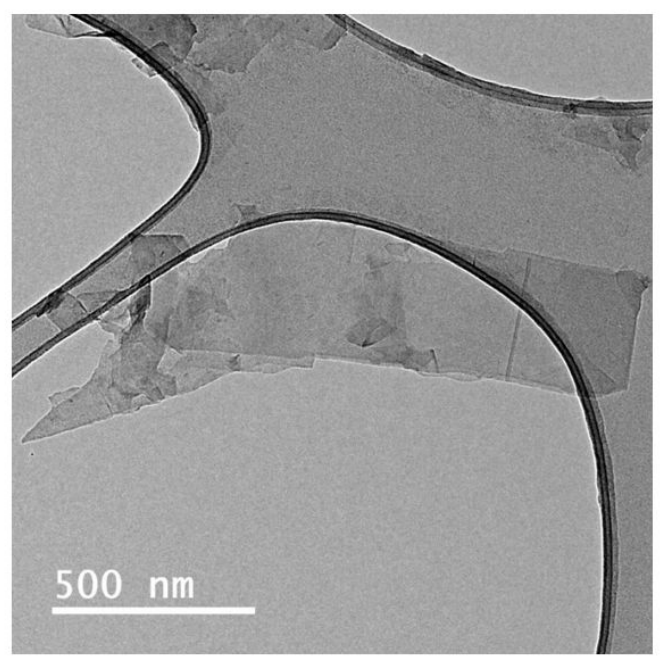

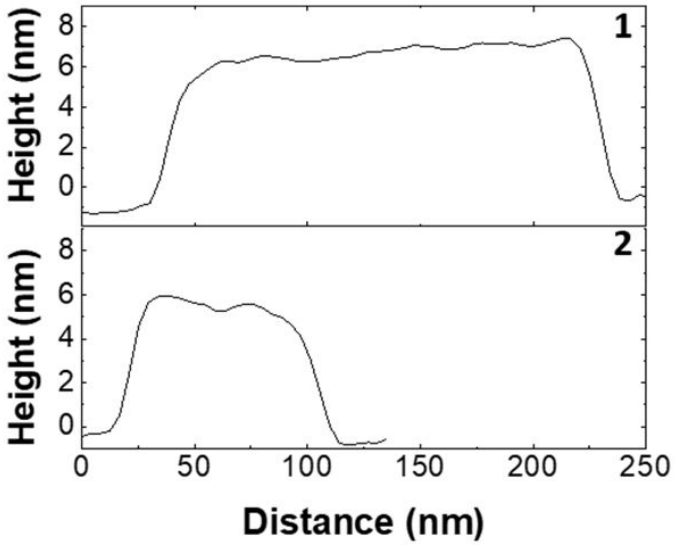
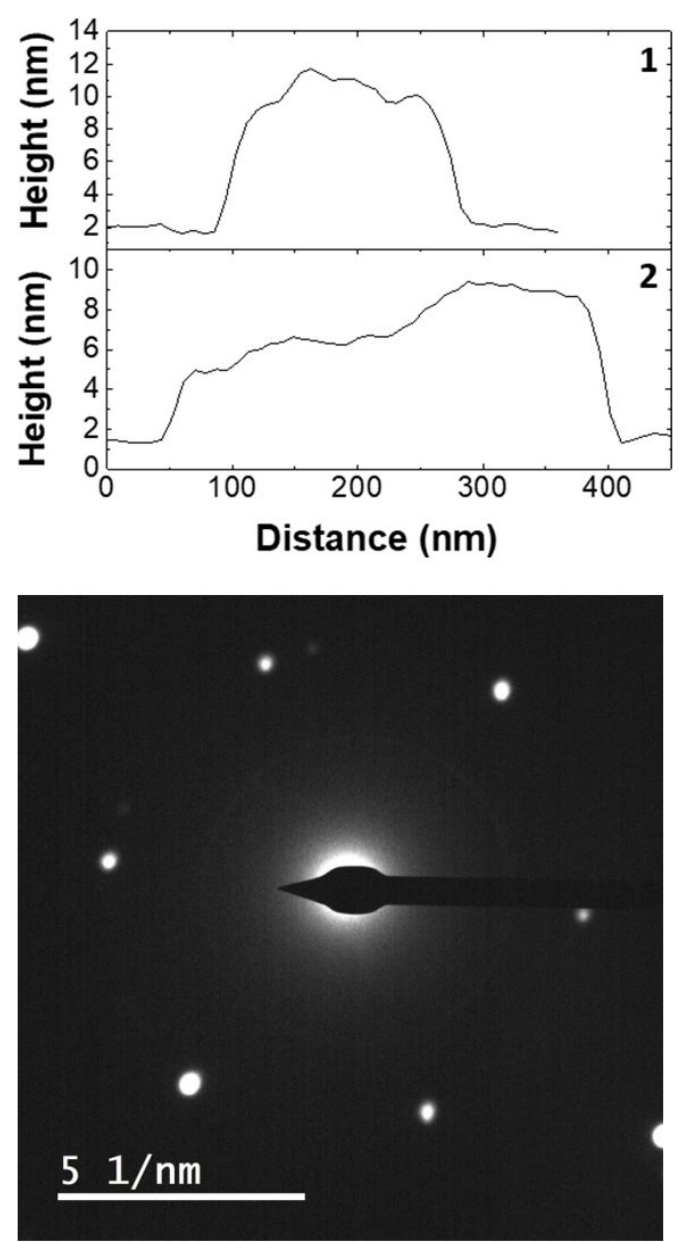

Figure 2 Representative AFM image and the corresponding height profile of selected graphene nanosheets of samples: (a) Gr/py-lys and (b) Gr/py-tau. (c) Representative Bright Field-TEM image and corresponding diffraction pattern of a representative graphene nanosheet of the $\mathrm{Gr} / \mathrm{py}$-lys sample.

Raman spectroscopy is a widely used technique for the characterization of graphene. ${ }^{47-49}$ The Raman spectrum of graphene shows two characteristic bands, the G- and 2D peaks, associated to the in-plane stretching vibration of $\mathrm{sp}^{2}$ carbons and to the breathing mode of the six-atom ring, respectively. ${ }^{50}$ Moreover, there is an additional characteristic band, the D-peak, which is activated by defects. ${ }^{47}$ In the case of mechanically exfoliated graphene, the shape of the 2D band can be used for identification of graphene. ${ }^{50} \mathrm{~A}$ similar approach can be used also for graphene produced by LPE. ${ }^{51-55}$ However, there are some important differences: the Raman spectrum of graphene produced by LPE is different from that of mechanically exfoliated graphene: first, a D-band is always present, activated by the edges, being the flakes of smaller size compared to the laser spot; second, the 2D band can show complex lineshapes due to solvent or stabiliser molecules adsorption, structural changes and/or re- 
stacking of flakes. ${ }^{48,56,57}$ Here we use a qualitative protocol based on the shape of the 2D peak, which was introduced in our previous works on characterisation of graphene produced by LPE. ${ }^{51-55}$ The protocol consists in fitting the 2D peak with single Lorentzian lineshape (Figure 1d). The quality of the fit, measured by the residual fit coefficient, allows distinguishing between single-layer graphene (SLG), few-layer graphene sheets (FLG) and thick graphitic layers ( $>10$ layers) with $A B$ stacking. Representative Raman spectra for each type of graphene layers are shown in Figure S5. Using this protocol and by measuring 50 isolated flakes, the percentage of SLG is $\sim 44 \%$ and $42 \%$ for $\mathrm{Gr} /$ py-lys and $\mathrm{Gr} /$ py-tau, respectively. This is in good agreement with the SLG content typically measured in graphene dispersions made by LPE, ( $30 \%$ of SLG), analysed using the same protocol in other works. ${ }^{51-55}$

To support AFM and Raman analysis, Gr/py-lys flakes were analysed by TEM. Representative TEM images of the graphene flakes are shown in Figure 2(c). TEM measurements showed several flakes with lateral size of a few hundred nanometers, in agreement with AFM measurements. Also, most of graphene flakes measured by TEM were observed to be SLG or FLG. More TEM images can be found in the Supporting Information.
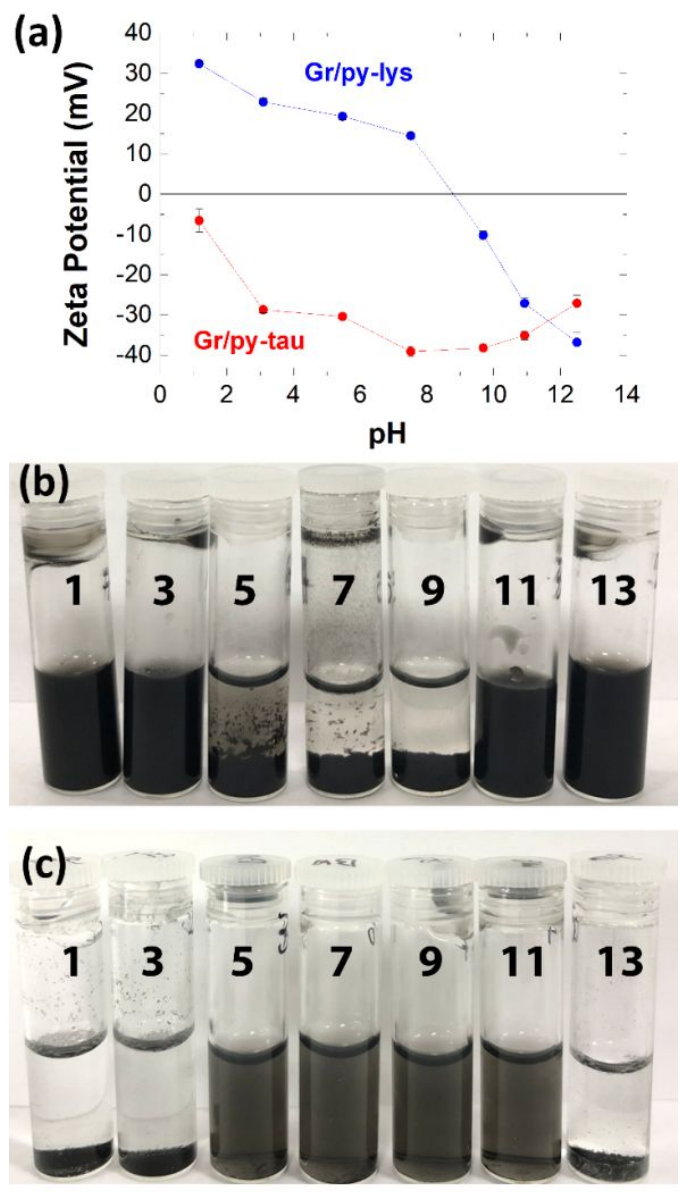

Figure 3 (a) Zeta potential measurements at different pH level. Photographs of the graphene dispersions at different pH level (pH 1 to pH 13 from left to right) after 1 day storage for (b) Gr/py-lys and (c) Gr/py-tau.

In order to change the surface charge of the graphene flakes in the solution, the $\mathrm{pH}$ of the as-prepared dispersions was systematically adjusted using concentrated $\mathrm{HCl}$ and $\mathrm{NaOH}$ solutions, starting from the as prepared dispersion ( $\left.\mathrm{pH}^{\sim}-4\right)$ and by increasing or decreasing the $\mathrm{pH}$. The change in graphene concentration was minimised by using minimal amount (less than $10 \%$ volume increase) of $\mathrm{HCl}$ and $\mathrm{NaOH}$ solutions. Figure $3(\mathrm{a})$ shows the zeta potential change while tuning the $\mathrm{pH}$ : Gr/py-lys solution shows strong decrease of zeta potential, with a change in its sign, from $32.4 \mathrm{mV}$ to $-36.8 \mathrm{mV}$ by increasing the $\mathrm{pH}$ from 1 to 13 . The zeta potential of $\mathrm{Gr} / \mathrm{py}$-tau solution stays negative throughout all $\mathrm{pH}$ level (from $-6.6 \mathrm{mV}$ to $-27.1 \mathrm{mV}$ by increasing the $\mathrm{pH}$ from 1 to 13). The difference in the behaviour of the two samples is attributed to their functional groups: py-lys with a carboxylic acid and a primary amine shows more sensitive response (i.e. changing its protonated state) to $\mathrm{pH}$ changes in the solution, whereas py-tau shows less sensitive response due to the strong tendency of the 
sulfonate group to stay deprotonated and the presence of a secondary amine group that allows the molecule to become neutral at low $\mathrm{pH}$ values by being protonated or negatively charged at higher ones by deprotonation. The stability of graphene dispersion is often measured by the absolute value of zeta potential, with benchmark stability values of $\pm 30 \mathrm{mV} .{ }^{58}$ As the zeta potential changes with the $\mathrm{pH}$, a strong change in the stability of the dispersion is expected. This is confirmed by visual inspection of the dispersions after one day from preparation. As shown in Figure 3(b) and (c), when the zeta potential of the dispersion is close to zero, the dispersion becomes unstable and stronger sedimentation is observed, e.g. at $\mathrm{pH}=7$ for $\mathrm{Gr} / \mathrm{py}$-lys and $\mathrm{pH}=1$ for $\mathrm{Gr} / \mathrm{py}$-tau. However, as soon as the zeta potential gets close to $\pm 30 \mathrm{mV}$, then stable dispersions are obtained. The optimum $\mathrm{pH}$ values, in term of stability, are $\mathrm{pH}=1$ and $\mathrm{pH}=13$ for $\mathrm{Gr} / \mathrm{py}$-lys and $\mathrm{pH}>5$ for $\mathrm{Gr} / \mathrm{py}$-tau. Note however that $\mathrm{Gr} / \mathrm{py}$-tau at $\mathrm{pH}=13$ showed lower zeta potential $(\zeta=-27.1 \mathrm{mV})$ and more sedimentation than at $\mathrm{pH}=9(\zeta=-38.2 \mathrm{mV})$ or $\mathrm{pH}=11(\zeta=-35.1 \mathrm{mV})$. This is due to the increased concentration of ionic species in the solution, which can lead to decreased electrostatic repulsion between the dispersed flakes, and therefore to re-stacking and sedimentation.

Finally, it is interesting to compare our results, where the $\mathrm{pH}$ is tuned after exfoliation, with the ones obtained by exfoliating graphite in a solution of py-lys and py-tau, already at different $\mathrm{pH}$. Thus, graphite was sonicated for 7 days in py-lys and py-tau solutions, prepared at three different $\mathrm{pH}$ levels: acidic $(\mathrm{pH} \approx 1)$, as-prepared and basic $(\mathrm{pH} \approx 12)$, following the same exfoliation protocol described in the experimental section. Figure S7 clearly shows that almost no exfoliation and complete sedimentation after just one day standing (even without centrifugation step) is obtained by exfoliation with both py-lys and py-tau solutions at extreme $\mathrm{pH}$ levels, in contrast to the results obtained by tuning the $\mathrm{pH}$ after exfoliation. This is because at extreme $\mathrm{pH}$, due to the high concentration of ionic species in the solution, the interaction between the pyrene molecules and graphene is disrupted. This observation is in agreement with previous studies by Schlierf et al., ${ }^{26}$ using pyrene functionalised with sulfonic groups, where it was observed that exfoliation of graphene is less effective at extreme $\mathrm{pH}$ due to the effect of ionic strength, inducing destabilisation. ${ }^{26}$

\section{Conclusion}

In this study, we have designed and produced pyrene-based stabilisers functionalised with amino acids for efficient production of aqueous graphene dispersions with amphoteric properties. Two types of pyrene derivatives were prepared and tested: pyrene-substituted lysine (py-lys) and taurine (py-tau). Both stabilisers were able to exfoliate graphite, producing dispersions containing good amounts of single-layer graphene. However, the use of py-lys as stabiliser gives rise to higher exfoliation efficiency by producing stable and relatively highly concentrated graphene dispersions. Furthermore, this stabiliser allows producing graphene dispersions with tunable charge by systematic change of the $\mathrm{pH}$ level, without producing sedimentation or destabilisation of the dispersed material at low or high $\mathrm{pH}$. In contrast, due to the very low $\mathrm{pKa}$ value of the sulfonate group in py-tau, stable dispersions were observed only for $\mathrm{pH}$ levels between 5 and 11 .

In conclusion, we have shown a simple approach that allows producing tunable surface charge graphene dispersions using simple stabilisers such as pyrene derivatives, functionalised with specific amino acids. As the amount and sign of the surface charge can be tuned by changing the $\mathrm{pH}$ of the solution, these dispersions offer a great potential for in vitro and in vivo studies.

\section{Acknowledgements}

We would like to thank the EPSRC for funding the 2D Health project (EP/P00119X/1). MZ acknowledges the Hewlett-Packard Company for financial support in the framework of the Graphene NowNano Doctoral Training Center. LHI thanks EPSRC for a PhD scholarship provided through the Doctoral Training Partnership scheme (grant EP/M507969/1).AB is supported by a research fellowship provided through the Dalton Nuclear Institute, the University of Manchester (Dalton Fellowship). Both LHI and AB acknowledge the support of The University of Manchester's Dalton Cumbrian Facility (DCF), a partner in the National Nuclear User Facility, the EPSRC UK National Ion Beam Centre and the Henry Royce Institute.

\section{References}

1. Novoselov, K. S. et al. Two-dimensional atomic crystals. Proc. Natl. Acad. Sci. 102, 10451-10453 (2005).

2. Novoselov, K. S. et al. A roadmap for graphene. Nature 490, 192-200 (2012).

3. Geim, A. K. \& Novoselov, K. S. The Rise of Graphene. 183-191 doi:10.1038/nmat1849

4. Hernandez, Y. et al. High yield production of graphene by liquid phase exfoliation of graphite. Nat. Nanotechnol. 3, 563-8 (2008). 
5. Coleman, J. N. Liquid-phase exfoliation of nanotubes and graphene. Adv. Funct. Mater. 19, 3680-3695 (2009).

6. Khan, U., O'Neill, A., Lotya, M., De, S. \& Coleman, J. N. High-concentration solvent exfoliation of graphene. Small 6, 864-871 (2010).

7. Nicolosi, V., Chhowalla, M., Kanatzidis, M. G., Strano, M. S. \& Coleman, J. N. Liquid Exfoliation of Layered Materials. Science (80-. ). 340, 1226419-1226419 (2013).

8. Xu, Q. Q., Zhao, W., Zhi, J. T. \& Yin, J. Z. Exfoliation of graphite in CO2 expanded organic solvent combined with low speed shear mixing. Carbon. 135, 180-186 (2018).

9. Paton, K. R. et al. Scalable production of large quantities of defect-free few-layer graphene by shear exfoliation in liquids. Nat. Mater. 13, 624-630 (2014).

10. Backes, C. et al. Guidelines for Exfoliation, Characterization and Processing of Layered Materials Produced by Liquid Exfoliation. Chem. Mater. 29, 243-255 (2017).

11. Karagiannidis, P. G. et al. Microfluidization of Graphite and Formulation of Graphene-Based Conductive Inks. ACS Nano 11, 2742-2755 (2017).

12. Zhang, Q. et al. Advanced review of graphene-based nanomaterials in drug delivery systems: Synthesis, modification, toxicity and application. Mater. Sci. Eng. C 77, 1363-1375 (2017).

13. Liu, Y., Dong, X. \& Chen, P. Biological and chemical sensors based on graphene materials. Chem. Soc. Rev. 41, 2283-2307 (2012).

14. Yoo, J. M., Kang, J. H. \& Hong, B. H. Graphene-based nanomaterials for versatile imaging studies. Chem. Soc. Rev. 44, 4835-4852 (2015).

15. Feng, L. \& Liu, Z. Graphene in biomedicine: opportunities and challenges. Nanomedicine 6, 317-324 (2011).

16. Yang, Y., Asiri, A. M., Tang, Z., Du, D. \& Lin, Y. Graphene based materials for biomedical applications. Mater. Today 16, 365-373 (2013).

17. Chung, C. et al. Biomedical Applications of Graphene and Graphene Oxide. Acc. Chem. Res. 46, 22112224 (2013).

18. Kostarelos, K. \& Novoselov, K. S. Exploring the Interface of Graphene and Biology. Science (80-. ). 344, (2014).

19. Hernandez, Y., Lotya, M., Rickard, D., Bergin, S. D. \& Coleman, J. N. Measurement of multicomponent solubility parameters for graphene facilitates solvent discovery. Langmuir 26, 3208-3213 (2010).

20. Gao, W. The Chemistry of Graphene Oxide. in Graphene Oxide 61-95 (Springer International Publishing, 2015). doi:10.1007/978-3-319-15500-5_3

21. Rodrigues, A. F. et al. A blueprint for the synthesis and characterisation of thin graphene oxide with controlled lateral dimensions for biomedicine. 2D Mater. 5, 035020 (2018).

22. Lotya, M. et al. Liquid phase production of graphene by exfoliation of graphite in surfactant/water solutions. J. Am. Chem. Soc. 131, 3611-3620 (2009).

23. Narayan, R., Lim, J., Jeon, T., Li, D. J. \& Kim, S. O. Perylene tetracarboxylate surfactant assisted liquid phase exfoliation of graphite into graphene nanosheets with facile re-dispersibility in aqueous/organic polar solvents. Carbon. 119, 555-568 (2017).

24. McManus, D. et al. Water-based and biocompatible 2D crystal inks for all-inkjet-printed heterostructures. Nat. Nanotechnol. 12, 1-19 (2017).

25. Parviz, D. et al. Dispersions of non-covalently functionalized graphene with minimal stabilizer. ACS Nano 6, 8857-8867 (2012).

26. Schlierf, A. et al. Nanoscale insight into the exfoliation mechanism of graphene with organic dyes: effect of charge, dipole and molecular structure. Nanoscale 5, 4205 (2013).

27. Yang, H. et al. A simple method for graphene production based on exfoliation of graphite in water using 1-pyrenesulfonic acid sodium salt. Carbon. 53, 357-365 (2013).

28. Lee, D. W., Kim, T. \& Lee, M. An amphiphilic pyrene sheet for selective functionalization of graphene. Chem. Commun. 47, 8259-8261 (2011).

29. Zhang, M. et al. Production of graphene sheets by direct dispersion with aromatic healing agents. Small 6, 1100-1107 (2010).

30. Bianco, A. Graphene: Safe or toxic? the two faces of the medal. Angew. Chemie - Int. Ed. 52, 49864997 (2013).

31. Fadeel, B. et al. Safety Assessment of Graphene-Based Materials: Focus on Human Health and the Environment. ACS Nano 12, 10582-10620 (2018).

32. Tu, Q. et al. Effects of surface charges of graphene oxide on neuronal outgrowth and branching. Analyst 139, 105-115 (2014). 
33. Lalwani, G., D’Agati, M., Khan, A. M. \& Sitharaman, B. Toxicology of graphene-based nanomaterials. Adv. Drug Deliv. Rev. 105, 109-144 (2016).

34. Zhou, T., Zhou, X. \& Xing, D. Controlled release of doxorubicin from graphene oxide based chargereversal nanocarrier. Biomaterials 35, 4185-4194 (2014).

35. Li, W., McManus, D., Liu, H., Casiraghi, C. \& Webb, S. J. Aqueous dispersions of nanostructures formed through the self-assembly of iminolipids with exchangeable hydrophobic termini. Phys. Chem. Chem. Phys. 19, 17036-17043 (2017).

36. Ahadian, S. et al. Facile and green production of aqueous graphene dispersions for biomedical applications. Nanoscale 7, 6436-6443 (2015).

37. Pattammattel, A. \& Kumar, C. V. Kitchen Chemistry 101: Multigram Production of High Quality Biographene in a Blender with Edible Proteins. Adv. Funct. Mater. 25, 7088-7098 (2015).

38. Joseph, D., Tyagi, N., Ghimire, A. \& Geckeler, K. E. A direct route towards preparing pH-sensitive graphene nanosheets with anti-cancer activity. RSC Adv. 4, 4085-4093 (2014).

39. Perkampus, H.-H. Analytical Applications of UV-VIS Spectroscopy. in UV-VIS Spectroscopy and Its Applications 26-80 (Springer Berlin Heidelberg, 1992). doi:10.1007/978-3-642-77477-5_4

40. Lotya, M., King, P. J., Khan, U., De, S. \& Coleman, J. N. High-concentration, surfactant-stabilized graphene dispersions. ACS Nano 4, 3155-3162 (2010).

41. Hunter, R. J. Zeta potential in colloid science : principles and applications. Colloid science 2, (1981).

42. Cheng, Z.-L., Cao, B.-C., Wu, P.-R., Ma, L. \& Liu, Z. Templated synthesis of graphene nanosheets within curling layered nanostructure of halloysite nanotubes. Mater. Lett. 202, 62-65 (2017).

43. Stigter, D. Micelle Formation by lonic Surfactants. II. Specificity of Head Groups, Micelle Structure. J. Phys. Chem. 78, 2480-2485 (1974).

44. Eklund, Gupta, A., Tadigadapa, S., Chen, G. \& Joshi, P. Raman Scattering from High-Frequency Phonons in Supported n -Graphene Layer Films. Nano Lett. 6, 2667-2673 (2006).

45. Nemes-Incze, P., Osváth, Z., Kamarás, K. \& Biró, L. P. Anomalies in thickness measurements of graphene and few layer graphite crystals by tapping mode atomic force microscopy. Carbon N. Y. 46, 1435-1442 (2008).

46. Backes, C. et al. Production of highly monolayer enriched dispersions of liquid-exfoliated nanosheets by liquid cascade centrifugation. ACS Nano 10, 1589-1601 (2016).

47. Ferrari, A. C. \& Basko, D. M. Raman spectroscopy as a versatile tool for studying the properties of graphene. 1-13 (2013). doi:10.1038/nnano.2013.46

48. Jorio, A., Saito, R., Dresselhaus, G. \& Dresselhaus, M. S. Raman Spectroscopy in Graphene Related Systems. Raman Spectroscopy in Graphene Related Systems (2011). doi:10.1002/9783527632695

49. Casiraghi, C. Raman spectroscopy of graphene. in Spectroscopic Properties of Inorganic and Organometallic Compounds: Techniques, Materials and Applications, Volume 43 29-56 (2012). doi:10.1039/9781849734899-00029

50. Ferrari, A. C. et al. Raman spectrum of graphene and graphene layers. Phys. Rev. Lett. 97, 1-4 (2006).

51. Ciesielski, A. et al. Harnessing the Liquid-Phase Exfoliation of Graphene Using Aliphatic Compounds: A Supramolecular Approach. Angew. Chemie Int. Ed. 53, 10355-10361 (2014).

52. Haar, S. et al. A supramolecular strategy to leverage the liquid-phase exfoliation of graphene in the presence of surfactants: Unraveling the role of the length of fatty acids. Small 11, 1691-1702 (2015).

53. Haar, S. et al. Enhancing the Liquid-Phase Exfoliation of Graphene in Organic Solvents upon Addition of n-Octylbenzene. Sci. Rep. 5, 16684 (2015).

54. Conti, S. et al. Perchlorination of Coronene Enhances its Propensity for Self-Assembly on Graphene. ChemPhysChem 17, 352-357 (2016).

55. Shin, Y. et al. Synthesis and characterization of composite membranes made of graphene and polymers of intrinsic microporosity. Carbon N. Y. 102, 357-366 (2016).

56. Kim, K. et al. Raman spectroscopy study of rotated double-layer graphene: Misorientation-angle dependence of electronic structure. Phys. Rev. Lett. 108, 1-6 (2012).

57. Eckmann, A. et al. Probing the nature of defects in graphene by Raman spectroscopy. Nano Lett. 12, 3925-3930 (2012).

58. Smith, R. J., Lotya, M. \& Coleman, J. N. The importance of repulsive potential barriers for the dispersion of graphene using surfactants. New J. Phys. 12, (2010). 